\title{
BMJ Open Drug use and torsades de pointes cardiac arrhythmias in Sweden: a nationwide register-based cohort study
}

\author{
Bengt Danielsson, ${ }^{1}$ Julius Collin, ${ }^{1}$ Anastasia Nyman, ${ }^{1}$ Annica Bergendal, ${ }^{1}$ \\ Natalia Borg, ${ }^{1}$ Maria State, ${ }^{1}$ Lennart Bergfeldt, ${ }^{2}$ Johan Fastbom (1) ${ }^{1}$
}

To cite: Danielsson B, Collin J, Nyman A, et al. Drug use and torsades de pointes cardiac arrhythmias in Sweden: a nationwide register-based cohort study. BMJ Open 2020;10:e034560. doi:10.1136/ bmjopen-2019-034560

- Prepublication history for this paper is available online. To view these files, please visit the journal online (http://dx.doi org/10.1136/bmjopen-2019034560).

Received 01 October 2019 Revised 30 January 2020 Accepted 12 February 2020

\section{Check for updates}

(c) Author(s) (or their employer(s)) 2020. Re-use permitted under CC BY-NC. No commercial re-use. See rights and permissions. Published by BMJ.

${ }^{1}$ Department of Analysis, Swedish National Board of Health and Welfare, Stockholm, Sweden

${ }^{2}$ Department of Molecular and Clinical Medicine, University of Gothenburg, Goteborg, Sweden

Correspondence to Dr Johan Fastbom; johan.fastbom@socialstyrelsen. se

\section{ABSTRACT}

Objective To study the occurrence of torsades de pointes (TdP) ventricular tachycardia in relation to use of drugs labelled with TdP risk, using two nationwide Swedish registers.

Design Prospective register-based cohort study. Setting Entire Sweden.

Participants Persons aged $\geq 18$ years prescribed and dispensed any drug classified with TdP risk during 2006-2017, according to CredibleMeds. Persons with a registered TdP diagnosis during the study period, using drugs labelled with known (TdP 1), possible (TdP 2) or conditional (TdP 3 ) risk at the incident of TdP were examined.

Primary outcome measures Occurrence of TdP in relation to exposure rates for individual drugs with $\mathrm{TdP}$ risk.

\section{Secondary outcome measures Concurrent use of} more than one TdP-labelled drug in a person with a TdP diagnosis.

Results During the study period, $410 \mathrm{TdP}$ cases using drugs with TdP risk labels at the incident were registered; 205 women and 205 men, mean age 74.0 and 71.5 years, respectively. Antidepressants dominated (129/410, 30\%), followed by antiarrhythmics (17\%). Diuretics and gastric acid-secretion inhibitors, with TdP risk related to induction of hypokalaemia or hypomagnesaemia, were used in $56 \%$ and $32 \%$ of the $410 \mathrm{TdP}$ cases, respectively. Among the most used antidepressants, citalopram with known TdP 1 risk was associated with both a higher absolute number and incidence of TdP per 100000 users (two to four times), compared with mirtazapine with possible (TdP 2), and sertraline with conditional (TdP 3 ) risk. Multiple risk factors, including advanced age, cardiovascular disease and treatment with more than one TdP-classified drug, were frequently observed.

Conclusions Antidepressants followed by antiarrhythmics dominated among TdP risk drugs used by adults with TdP diagnosis, the majority being $\geq 65$ years. TdP risk class and concomitant medication should be considered when prescribing antidepressants to older patients.

\section{INTRODUCTION}

Torsades de pointes (TdP) ventricular tachycardia is a rare but life-threatening condition that can deteriorate into ventricular fibrillation and cardiac arrest and, in the worst case

\section{Strengths and limitations of this study}

This study used individual-based data from nationwide registers on inpatient diagnoses and drug dispensations from pharmacies, making it possible to estimate the incidence of TdP per 100000 users of prescribed individual drugs labelled with torsades de pointes (TdP) risk.

- To our knowledge, the study is, so far, the largest in terms of number of patients with TdP diagnosis.

- One limitation is that the data on drug use do not include information about drug treatment at hospitals.

scenario, sudden cardiac death. Symptoms may, however, vary from dizziness, syncope and seizures to cardiac arrest/sudden death. Prolongation of the corrected QT interval (QTc) on the ECG may predispose to TdP, together known as long QT syndrome (LQTS). LQTS can be congenital or acquired. ${ }^{12}$ Drugs in most therapeutic groups, including several widely used antidepressant and antipsychotic drugs, have been shown to be associated with an increased risk of acquired LQTS, mainly due to blockade of one of the cardiac potassium channels expressed by the hERG gene. This results in inhibition of a major repolarising potassium current, IKr. According to the CredibleMeds classification system, ${ }^{3}$ which is updated regularly by an expert panel, drugs that can prolong the QTc interval have different propensities to cause TdP (see the Methods section).

During the past decades, safety signals for QTc prolongation and TdP have resulted in the withdrawal from the market of some psychotropic drugs that block the hERG channel (eg, sertindole or thioridazine ${ }^{4}$ ) or a restriction in their use (eg, citalopram ${ }^{5} 6$ and haloperidol ${ }^{7}$ ) in many countries. Several studies also indicate that the risk of druginduced $\mathrm{TdP}$ is particularly relevant in combination with other patient-specific risk factors, such as age, cardiovascular diseases, 
electrolyte disturbances or simultaneous use of drugs increasing the risk of TdP. ${ }^{348-12}$

Previous studies have indicated that TdP is underreported. One reason is that accurate diagnosis requires ECG recording during the event. Another reason is that a sizeable proportion of patients with TdP will not survive the ventricular arrhythmia. All-cause death, adjusted for a number of potential comorbidity confounders, has therefore been used as a proxy for studying risks of using TdPclassified antipsychotics ${ }^{13-15}$ or antidepressants ${ }^{16}$ in the elderly.

Case report studies provide unique information about the link between TdP-classified drugs and TdP. However, until now, these studies have been relatively small and have not allowed comparisons of TdP risk between drugs, as they have not taken drug exposure rates into account. ${ }^{9}$ Therefore the main objective of this study, based on Swedish national registries, was to investigate occurrence of $\mathrm{TdP}$ in relation to exposure rates (incidence of $\mathrm{TdP}$ per 100000 users of a TdP-classified drug) for individual drugs with TdP risk, in persons aged $18-64$ and $\geq 65$ years. The second aim was to study the concomitant use of more than one TdP-labelled drug in a person with a TdP diagnosis.

\section{METHODS}

\section{Study population and registers}

This was a cohort study. The study population comprised all individuals above the age of 18 years who had been prescribed and dispensed any drug classified with TdP risk according to CredibleMeds, from 1 January 2006 to 31 December 2017. The study was based on data from two Swedish registers linked by the personal identification number: the Swedish Patient Register (SPR) and the Swedish Prescribed Drug Register (SPDR). These registers are administered by the Swedish National Board of Health and Welfare, an authority under the Swedish Ministry of Health and Social Affairs. The collection of register data is governed by Swedish legislation, and it is mandatory for Swedish healthcare and pharmacies to report the data. Information on the outcome, first-time $\mathrm{TdP}$ diagnosis (International Classification of Diseases, 10th Revision (ICD-10) code I47.2), was retrieved from the SPR, which covers all inpatient and specialised outpatient care in Sweden. ${ }^{18}$ Drug exposure data were retrieved from SPDR, which has detailed individual-based information about all prescribed and dispensed drugs at Swedish pharmacies. ${ }^{19}$

\section{Exposure to drugs labelled with QTc prolongation/TdP risk}

Prescription data were collected for the time period of 90 days before the reported TdP event (from hereon named index event). For each prescribed drug, the duration of the treatment was calculated by dividing the amount of dispensed drug with the prescribed daily dose ${ }^{19}$ In order to ensure relevant exposure, only prescriptions lasting until or beyond the index event date were included in the analyses. In the following text and tables, this is labelled as 'use'. All drugs classified with risk of TdP according to CredibleMeds ${ }^{3}$ (http://www.crediblemeds.org, 31 October 2018) were examined: TdP $1=$ known risk of $\mathrm{TdP}$ (list 1), TdP 2=possible risk of TdP (list 2) and TdP $3=$ conditional risk of TdP (list 3).

The number of users of individual drugs within a certain therapeutic group varies considerably, as well as between various age groups. We therefore estimated the incidence of TdP per 100000 users of individual TdP-labelled drugs in persons aged $\geq 65$ years (definition of older adults in Sweden) and in individuals aged 18-64 years, during the whole study period. Here a user of a drug was defined as a person to whom the pharmacy had dispensed the drug at least once during the study period.

\section{Additional risk factors for TdP}

As mentioned in the Introduction section, several case report studies suggest that the risk of drug-induced TdP is particularly relevant in combination with other important patient-specific risk factors, in particular genetic susceptibility (LQTS mutations), cardiovascular disease, electrolyte disturbances (hypokalaemia and hypomagnesaemia), or simultaneous use of two or more drugs which increase the risk of TdP. ${ }^{34-10}$ By using data from SPDR, it has been possible to study the occurrence of some major established contributing risk factors, in each individual at the incident of TdP, as follows: (1) concomitant use of more than one TdP-classified drug, (2) treatment with diuretics which may cause hypokalaemia and hypomagnesaemia, (3) treatment with acid-secretion inhibitors (proton pump inhibitors and $\mathrm{H}_{2}$-receptor antagonists) which may cause hypomagnesaemia and (4) use of drugs against cardiovascular disease (Anatomical, Therapeutic, Chemical (ATC) code C).

\section{PATIENT AND PUBLIC INVOLVEMENT (PPI)}

This study was based on data from Swedish healthcare registers. We did not directly include PPI in this study, but the registers used in the study are developed with PPI and updated by a committee that includes patient representatives.

\section{RESULTS \\ Overview: TdP diagnosis and use of TdP-labelled drugs}

There were 410 persons registered with TdP diagnosis using drugs with TdP risk labels at the TdP incident: 205 women and 205 men, with a mean age of 74.0 and 71.5 years, respectively.

\section{$\mathrm{TdP}$ in relation to TdP risk category for drugs blocking the $\mathrm{hERG}$ channel}

As shown in table 1, drugs blocking the cardiac hERG channel and labelled TdP 1 in normal use (ie, when used as directed according to the Summary of Product Characteristics) were used more often at the index event 
Table 1 Use ${ }^{*}$ of TdP risk classified drugs at the event of TdP, in relation to therapeutic group and TdP risk class (acidsecretion drugs and diuretics with TdP 3 risk classification but not blocking the hERG channel excluded), expressed as number of TdP cases when a certain drug was used* during the study period 2006-2017

\begin{tabular}{|c|c|c|c|c|c|c|c|c|c|c|}
\hline \multirow{2}{*}{\multicolumn{2}{|c|}{$\begin{array}{l}\begin{array}{l}\text { Antidepressant } \\
\text { drugs }\end{array} \\
\text { TdP } 1\end{array}$}} & \multicolumn{2}{|l|}{ Antipsychotic drugs } & \multicolumn{2}{|c|}{ Antinfective drugs } & \multicolumn{2}{|l|}{$\begin{array}{l}\text { Cardiovascular } \\
\text { drugs }\end{array}$} & \multicolumn{2}{|l|}{ Drugs in other groups } & \multirow{2}{*}{$\begin{array}{l}\text { Total } \\
175\end{array}$} \\
\hline & & TdP 1 & & TdP 1 & & TdP 1 & & TdP 1 & & \\
\hline Citalopram & 72 & Haloperidol & 3 & Ciprofloxacin & 4 & Amiodarone & 19 & Donepezil & 7 & \\
\hline \multirow[t]{4}{*}{ Escitalopram } & 5 & Levomepromazine & 2 & Fluconazole & 2 & Dronedarone & 3 & Methadone & 4 & \\
\hline & & & & Moxifloxacin & 2 & Disopyramide & 9 & Papaverine & 2 & \\
\hline & & & & Erytromycin & 1 & Flecainide & 3 & & & \\
\hline & & & & & & Sotalol & 37 & & & \\
\hline TdP 2 & & TdP 2 & & TdP 2 & & TdP 2 & & TdP 2 & & 80 \\
\hline Mirtazapine & 28 & Olanzapine & 9 & & & & & Alfuzosin & 13 & \\
\hline Clomipramine & 5 & Aripiprazole & 1 & & & & & Tolterodine & 5 & \\
\hline \multirow[t]{6}{*}{ Venlafaxine } & 2 & Clozapine & 1 & & & & & Solifenacin & 3 & \\
\hline & & Litium & 1 & & & & & Memantine & 3 & \\
\hline & & Perphenazine & 1 & & & & & Buprenorphine & 2 & \\
\hline & & Risperidone & 1 & & & & & Promethazine & 2 & \\
\hline & & & & & & & & Tacrolimus & 2 & \\
\hline & & & & & & & & Tamoxifen & 1 & \\
\hline TdP 3 & & TdP 3 & & TdP 3 & & TdP 3 & & TdP 3 & & 66 \\
\hline Sertraline & 14 & Quetiapine & 3 & Posaconazole & 1 & Isradipine & 3 & Hydroxizine & 20 & \\
\hline Amitriptylin & 5 & & & Metronidazole & 1 & & & Metoclopramide & 6 & \\
\hline Fluoxetine & 3 & & & & & & & Loperamide & 5 & \\
\hline Paroxetine & 2 & & & & & & & Hydroxychloroquine & 3 & \\
\hline Total & 136 & & 22 & & 11 & & 74 & & 78 & 321 \\
\hline
\end{tabular}

*A person is considered to have used a drug if the person has a dispensed prescription lasting until or beyond the TdP event day. See the Methods section for details.

TdP, torsades de pointes.

than drugs classified as TdP 2 or TdP 3. Among various therapeutic classes, antidepressants were predominantly used in the TdP cases, followed by antiarrhythmic drugs. Several cases were also using antipsychotic, antibiotic/ antimycotic and urological drugs. Individual drugs in other therapeutic drug groups, used by four or more persons at the index event, included the Alzheimer drug donepezil, the analgesic methadone, the anxiolytic drug hydroxyzine, and the gastrointestinal drugs loperamide and metoclopramide.

\section{TdP-classified drugs not blocking the hERG channel}

Almost all diuretics (except a few potassium saving diuretics) and all acid-secretion inhibitors are classified as TdP 3 due to their potential to induce potassium deficiency (diuretics) and magnesium deficiency (diuretics and acid-secretion inhibitors), promoting the triggering of TdP. Both are known risk factors for development of $\mathrm{TdP}$, especially when the person simultaneously uses a hERG-blocking drug. Our results show that 229 out of 410 cases $(56 \%)$ with TdP diagnosis had used TdP 3-labelled diuretics and 131 (32\%) TdP 3-labelled acid-secretion inhibitors.

Detailed information on use of TdP-classified drugs-alone or in combination-at the TdP event

Tables 2 and 3 show the absolute number and estimated incidence of TdPs per 100000 users of each identified TdP-classified hERG-blocking drug used at the index event in persons aged $18-64$ and $\geq 65$ years. In tables 4 and 5 , information on the use of additional TdP-classified drugs in various $\mathrm{TdP}$ categories at the index event (at least one additional drug, any therapeutic class) is presented. In many TdP cases, several TdP-classified drugs had been used simultaneously. The highest number was six in three index cases.

\section{Antidepressant drugs}

In total, 125 persons used TdP risk classified antidepressants (in total 136 prescriptions with 9 different antidepressants; see tables 1 and 2), that is, $30 \%$ of all TdPs (125 out of $410 \mathrm{TdP}$ cases). Table 1 also shows that the TdP 1-classified drugs (citalopram and escitalopram) 
Table 2 Estimated incidence of TdP among users* of specific TdP-classified antidepressants, antipsychotics or other central nervous system active drugs, in patients aged $\geq 65$ and 18-64 years during 2006-2017 (the number of TdP cases using the specific drug is shown in parentheses)

\begin{tabular}{|c|c|c|c|c|c|}
\hline $\begin{array}{l}\text { Therapeutic group and } \\
\text { specific drug }\end{array}$ & $\begin{array}{l}\text { TdP } \\
\text { class }\end{array}$ & $\begin{array}{l}\text { TdP per } 100000 \\
\geq 65 \text { years (number } \\
\text { of TdP cases) }\end{array}$ & $\begin{array}{l}\text { Number of users } \\
\geq 65 \text { years }\end{array}$ & $\begin{array}{l}\text { TdP per } 100000 \\
\text { 18-64 years } \\
\text { (number of TdP cases) }\end{array}$ & $\begin{array}{l}\text { Number of users } \\
18-64 \text { years }\end{array}$ \\
\hline \multicolumn{6}{|l|}{ Antidepressant drugs } \\
\hline Citalopram & 1 & $15.3(59)$ & 385828 & $2.7(13)$ & 491640 \\
\hline Escitalopram & 1 & $4.1(3)$ & 73769 & $0.7(2)$ & 267733 \\
\hline Venlafaxine & 2 & $3.7(2)$ & 54392 & 0 & 225566 \\
\hline Mirtazapine & 2 & $7.5(23)$ & 307734 & $1.2(5)$ & 402370 \\
\hline Sertraline & 3 & $5.2(9)$ & 172728 & $0.9(5)$ & 588495 \\
\hline Paroxetine & 3 & $8.7(2)$ & 23057 & 0 & 74337 \\
\hline Fluoxetine & 3 & $4.8(1)$ & 21026 & 0 & 158495 \\
\hline Clomipramine & 3 & $13.2(2)$ & 15181 & $7.4(3)$ & 40364 \\
\hline Amitriptyline & 3 & $3.2(5)$ & 145624 & 0 & 280218 \\
\hline \multicolumn{6}{|l|}{ Antipsychotic drugs } \\
\hline Haloperidol & 1 & $3.1(2)$ & 63815 & $4.4(1)$ & 22730 \\
\hline Levomepromazine & 1 & 0 & 18355 & $4.0(2)$ & 49666 \\
\hline Olanzapine & 2 & $6.9(2)$ & 29128 & $7.4(7)$ & 94560 \\
\hline Aripiprazole & 2 & 0 & 5546 & $2.2(1)$ & 46073 \\
\hline Clozapine & 2 & 0 & 3420 & $11.1(1)$ & 8661 \\
\hline Lithium & 2 & 0 & 12098 & $2.6(1)$ & 38398 \\
\hline Perphenazine & 2 & $19.2(1)$ & 5208 & 0 & 11164 \\
\hline Risperidone & 2 & $0.9(1)$ & 110645 & 0 & 51641 \\
\hline Quetiapine & 3 & 0 & 22668 & $3.0(3)$ & 98819 \\
\hline \multicolumn{6}{|l|}{ Anti-dementia drugs } \\
\hline Donepezil & 1 & $8.1(7)$ & 86426 & 0 & 2487 \\
\hline Memantine & 3 & $4.7(3)$ & 64135 & 0 & 3047 \\
\hline \multicolumn{6}{|l|}{ Opioid analgesics } \\
\hline Methadone & 1 & 0 & 5411 & $41.9(4)$ & 9553 \\
\hline Buprenorphine & 2 & $0.6(1)$ & 170943 & $2.9(1)$ & 51603 \\
\hline \multicolumn{6}{|l|}{ Anxiolytic drugs } \\
\hline Hydroxyzine & 3 & $4.9(14)$ & 288469 & $0.7(6)$ & 913194 \\
\hline Promethazine & 3 & 0 & 48370 & $0.6(2)$ & 316428 \\
\hline
\end{tabular}

${ }^{*}$ A person is considered to have used a drug if the person has a dispensed prescription lasting until or beyond the TdP event day. See the Methods section for details.

TdP, torsades de pointes.

dominated, with 77 out of 136 prescriptions (57\%) of antidepressant drugs used at the time of the TdP. Corresponding figures for TdP 2-classified and TdP 3-classified antidepressants were $26 \%$ and $18 \%$.

The number and estimated incidence of TdP for individual antidepressant drugs is presented in table 2 . Both the number and the incidence of $\mathrm{TdP}$ with the three most prescribed antidepressants (citalopram, mirtazapine and sertraline) were five to six times higher in individuals aged $\geq 65$ years than in younger patients (table 2). The TdP risk for the three most used antidepressants among the older patients agreed with their TdP risk class. The TdP 1-classified citalopram had the highest absolute number of TdP (59 persons) and incidence of TdP (15/100000), followed by TdP 2-classified mirtazapine (23 TdPs, incidence of 7.5/100000) and TdP 3-classified sertraline (9 TdPs, incidence of 5.2/100000). A similar trend was observed in younger persons, however, with much lower absolute numbers and incidences compared with older persons (table 2).

The great majority (73\%, 91 of 125) of TdP cases who used TdP-classified antidepressant drugs also used at least one additional TdP-classified drug at the index event. TdP 3-classified diuretics $(42 \%, 53 / 125)$ and 
Table 3 Estimated incidence of TdP among users ${ }^{*}$ of specific TdP-classified antiarrhythmic, antihypertensive, anti-infective, urology, gastrointestinal, cancer or antirheumatic drugs in patients aged $\geq 65$ and 18-64 years during 2006-2017 (the number of TdP cases using the specific drug are shown in parentheses)

\begin{tabular}{|c|c|c|c|c|c|}
\hline $\begin{array}{l}\text { Therapeutic group and } \\
\text { specific drug }\end{array}$ & $\begin{array}{l}\text { TdP } \\
\text { class }\end{array}$ & $\begin{array}{l}\text { TdP per } 100000 \\
\geq 65 \text { years } \\
\text { (number of TdP cases) }\end{array}$ & $\begin{array}{l}\text { Number of } \\
\text { users } \geq 65 \\
\text { years }\end{array}$ & $\begin{array}{l}\text { TdP per } 100000 \\
18-64 \text { years } \\
\text { (number of TdP cases) }\end{array}$ & $\begin{array}{l}\text { Number of } \\
\text { users 18- } \\
64 \text { years }\end{array}$ \\
\hline \multicolumn{6}{|l|}{ Antiarrhythmic drugs } \\
\hline Disopyramide & 1 & $231.2(9)$ & 3893 & 0 & 1993 \\
\hline Sotalol & 1 & $81.1(31)$ & 38203 & $41.2(6)$ & 14547 \\
\hline Amiodarone & 1 & $61.3(13)$ & 21201 & $66.5(6)$ & 9029 \\
\hline Flecainide & 1 & $23.3(2)$ & 8566 & $10.2(1)$ & 9842 \\
\hline Dronedarone & 1 & $9.5(1)$ & 10552 & $33.2(2)$ & 5944 \\
\hline \multicolumn{6}{|l|}{ Antihypertensive drugs } \\
\hline Isradipine & 3 & $33.1(3)$ & 9061 & 0 & 3846 \\
\hline \multicolumn{6}{|l|}{ Antinfective drugs } \\
\hline Erythromycin & 1 & 0 & 71884 & $0.3(1)$ & 320592 \\
\hline Ciprofloxacin & 1 & $0.5(3)$ & 589531 & $0.1(1)$ & 703926 \\
\hline Moxifloxacin & 1 & $15.1(2)$ & 13190 & 0 & 21572 \\
\hline Flukonazole & 1 & $1.2(2)$ & 165084 & 0 & 471278 \\
\hline Posaconazole & 3 & $132(1)$ & 756 & 0 & 1505 \\
\hline Metronidazole & 3 & 0 & 204846 & $0.2(1)$ & 592134 \\
\hline \multicolumn{6}{|l|}{ Drugs in urology } \\
\hline Alfuzosin & 2 & $4.6(11)$ & 239618 & $1.5(2)$ & 136139 \\
\hline Solifenacin & 3 & $2.5(2)$ & 80771 & $2.3(1)$ & 42623 \\
\hline Tolterodine & 3 & $3.4(4)$ & 118155 & $1.9(1)$ & 52520 \\
\hline \multicolumn{6}{|l|}{ Gastrointestinal drugs } \\
\hline Papaverine & 1 & $1.8(1)$ & 54797 & $0.9(1)$ & 115111 \\
\hline Metoclopramide & 3 & $1.7(4)$ & 237601 & $0.7(2)$ & 273331 \\
\hline Loperamide & 3 & $1.6(3)$ & 189086 & $1.2(2)$ & 160901 \\
\hline \multicolumn{6}{|l|}{ Drugs in cancer } \\
\hline Tamoxifen & 2 & $2.9(1)$ & 34252 & 0 & 32127 \\
\hline Tacrolimus & 2 & 0 & 2845 & $24.8(2)$ & 2845 \\
\hline \multicolumn{6}{|l|}{ Antireumatic drug } \\
\hline Hydoxychloroquine & 3 & $21.6(2)$ & 9247 & $5.0(1)$ & 19929 \\
\hline
\end{tabular}

${ }^{*} \mathrm{~A}$ person is considered to have used a drug if the person has a dispensed prescription lasting until or beyond the TdP event day. See the Methods section for details.

TdP, torsades de pointes.

gastric acid-secretion inhibitors $(29 \%, 36 / 125)$ were the most common. However, concurrent use of drugs with known (TdP 1) or possible ( TdP 2) risk was also relatively common with other therapeutic classes, including antipsychotics, urological drugs, antidementia drugs and antiarrhythmic drugs (see table 4).

Antiarrhythmic drugs

In total, 71 persons $(17 \%)$ with a TdP diagnosis used class III antiarrhythmic drugs classified with TdP risk (tables 1 and 3). These drugs showed the highest incidence of $\mathrm{TdP}$ diagnoses per 100000 users, compared with drugs in other therapeutic classes, in all age groups (table 3). The highest TdP risk was observed for disopyramide in elderly persons, with a calculated incidence of 231/100000 users. Our results (cf tables 4 and 5) suggest a lower use of additional TdP 1 and TdP-classified drugs when TdPclassified antiarrhythmic drug were used, compared with when classified antidepressants and antipsychotics were used. However, additional TdP 3-classified drugs (mainly diuretics and acid-secretion inhibitors) were used by the majority of $\mathrm{TdP}$ cases treated with antiarrhythmics (exception: disopyramide and sotalol).

Antipsychotic drugs and other central nervous system (CNS) active drugs

The majority $(10 / 18)$ of reported TdP cases on antipsychotics were observed in persons younger than 65 years 
Table 4 Use* of TdP risk classified drugs (at least one, any therapeutic group) in addition to a specific TdP-classified antidepressant, antipsychotic, antidementia, opioid or anxiolytic drug, expressed as number of TdP cases using a specific TdP-classified drug (proportions (\%) using an additional TdP-classified drug are shown in parentheses)

\begin{tabular}{|c|c|c|c|c|c|c|}
\hline $\begin{array}{l}\text { Therapeutic group and } \\
\text { specific drug }\end{array}$ & $\begin{array}{l}\text { TdP } \\
\text { class }\end{array}$ & $\mathbf{n}$ & $\begin{array}{l}\text { Use of other } \\
\text { TdP } 1 \text { drugs }\end{array}$ & $\begin{array}{l}\text { Use of other TdP } 2 \\
\text { drugs }\end{array}$ & $\begin{array}{l}\text { Use of other TdP } 3 \\
\text { drugs }\end{array}$ & $\begin{array}{l}\text { Use of other TdP 1, } 2 \text { or } \\
3 \text { drugs }\end{array}$ \\
\hline \multicolumn{7}{|l|}{ Antidepressant drugs } \\
\hline Escitalopram & 1 & 5 & 0 & $2 / 5(40)$ & $2 / 5(40)$ & $4 / 5(80)$ \\
\hline Venlafaxine & 2 & 2 & $1 / 2(50)$ & $1 / 2(50)$ & $1 / 2(50)$ & $2 / 2(100)$ \\
\hline Fluoxetine & 3 & 3 & 0 & 1/3 (33) & 2/3 (67) & 2/3 (67) \\
\hline Paroxetine & 3 & 2 & $1 / 2(50)$ & $1 / 2(50)$ & $1 / 2(50)$ & 2/2 (100) \\
\hline Sertraline & 3 & 14 & 2/14 (14) & $3 / 14(21)$ & $10 / 14(71)$ & $11 / 14(79)$ \\
\hline Amitriptyline & 3 & 5 & $2 / 5(40)$ & $1 / 5(20)$ & $3 / 5(60)$ & $3 / 5(60)$ \\
\hline \multicolumn{7}{|l|}{ Antipsychotic drugs } \\
\hline Olanzapine & 2 & 9 & 2/9 (22) & $5 / 9(56)$ & $5 / 9(56)$ & $6 / 9(67)$ \\
\hline Aripiprazole & 2 & 1 & 0 & $1 / 1(100)$ & $1 / 1(100)$ & $1 / 1(100)$ \\
\hline Clozapine & 2 & 1 & 0 & $1 / 1(100)$ & $1 / 1(100)$ & $1 / 1(100)$ \\
\hline Lithium & 2 & 1 & 0 & $1 / 1(100)$ & $1 / 1(100)$ & $1 / 1(100)$ \\
\hline Perphenazine & 2 & 1 & 0 & 0 & $1 / 1(100)$ & $1 / 1(100)$ \\
\hline Risperidone & 2 & 1 & $1 / 1(100)$ & $1 / 1(100)$ & 0 & $1 / 1(100)$ \\
\hline Quetiapine & 2 & 3 & 2/3 (67) & 1/3 (33) & 2/3 (67) & $3 / 3(100)$ \\
\hline \multicolumn{7}{|l|}{ Antidementia drugs } \\
\hline Donepezil & 1 & 7 & $3 / 7(43)$ & 2/7 (29) & $5 / 7(71)$ & $5 / 7(71)$ \\
\hline Hydroxyzine & 3 & 20 & $6 / 20(30)$ & $9 / 20(45)$ & $15 / 20(75)$ & 19/20 (95) \\
\hline
\end{tabular}

${ }^{*}$ A person is considered to have used a drug if the person has a dispensed prescription lasting until or beyond the TdP event day. See the Methods section for details.

TdP, torsades de pointes.

(table 2). Combined use of TdP-classified antipsychotics and/or antidepressants was observed in the majority of persons using antipsychotics at the index event. Use of the opioids methadone and buprenorphine, the antidementia drugs donepezil and memantine, and the anxiolytic drugs hydroxyzine were also associated with TdP (table 1).

Drugs against infections, urological disorders and other diseases The incidence of TdP per 100000 users was low for commonly used antibiotics and fungicides. Twenty-one persons used TdP 2-classified urological drugs (alfuzosin, tolterodine or solifenacin) at the index event (tables 1 and 3). In most cases, other TdP-classified drugs were used concomitantly (table 5). Moreover, some TdP-classified drugs against cancer, rheumatic disease and gastrointestinal disorders were used at the index event (table 3). In all these TdP cases except for tamoxifen, at least two TdP-classified drugs were used at the event (table 5).

\section{TdP diagnosis and cardiovascular disease}

Treatment with cardiovascular drugs was very common among the $410 \mathrm{TdP}$ cases, $85 \%$ (348) in all ages and $88 \%$ $(286 / 326)$ in persons aged $\geq 65$ years.

\section{DISCUSSION}

The main findings of this register-based nationwide cohort study of 410 cases using TdP risk classified drugs, 
Table 5 Use* of TdP risk classified drugs (at least one, any therapeutic group) in addition to a specific TdP-classified antiarrhythmic, antihypertensive, anti-infective, urology, gastrointestinal, cancer or antirheumatic drug expressed as the number of TdP cases using a specific TdP-classified drug (proportions (\%) using an additional TdP-classified drug are shown in parentheses)

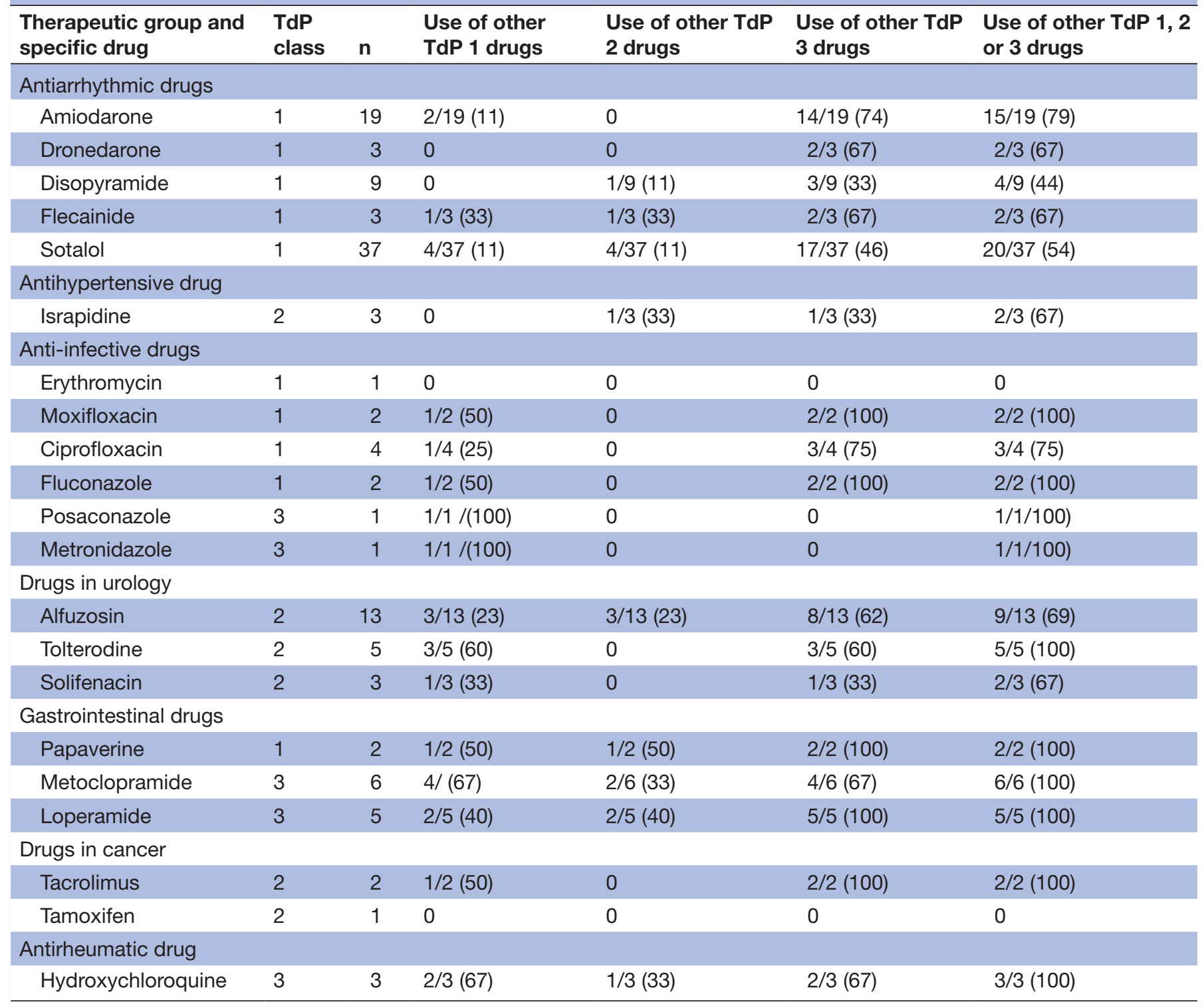

${ }^{*}$ A person is considered to have used a drug if the person has a dispensed prescription lasting until or beyond the TdP event day. See the Methods section for details.

TdP, torsades de pointes.

with a recorded TdP diagnosis, during 2006-2017, applying risk classification according to CredibleMeds, were (1) the vast majority of TdP cases were older than 65 years; (2) antidepressant drugs were the most common TdP-classified drugs used at the index event, followed by antiarrhythmic drugs; and (3) concomitant use of more than one TdP risk classified drug was very common, as was use of drugs for cardiovascular diseases. Because a diagnosis of TdP can only be ascertained in a living person and sudden cardiac death is one possible outcome of a $\mathrm{TdP}$, we assume that this patient cohort represents only the tip of an iceberg.

\section{Multiple risk factors common in TdP}

The present study showed that $\mathrm{TdP}$ is much more common in the elderly than in the younger age groups. For the most used antidepressant drugs (citalopram, mirtazapine and sertraline), the incidence of $\mathrm{TdP}$ was five to six times higher in persons aged $\geq 65$ years than in younger persons. Advanced age is a recognised risk factor for the development of TdP associated with the use of QTc-prolonging drugs. ${ }^{1411} 12$ Ageing is also associated with polypharmacy-in Sweden, people aged $\geq 75$ years are prescribed on average five different drugs ${ }^{20}$ and there is comprehensive evidence that concomitant 
administration of more than one TdP-classified drug further increases the risk of developing a QTc prolongation and TdP. ${ }^{3421-25}$ Our results are confirmative in this aspect. The most common of the additional drugs were diuretics and acid-secretion inhibitors. These drugs lack hERG-blocking potential but are classified with conditional risk (TdP 3 risk) due to their potential to cause hypokalaemia and hypomagnesaemia, respectively. These results agree with recent studies indicating that electrolyte changes play an important contributing role in the development of TdP. ${ }^{11}{ }^{12} 26$ Furthermore, a high proportion (almost $90 \%$ ) of elderly persons treated with TdPclassified drugs at the index event were also treated with drugs against cardiovascular disease, supporting that cardiovascular disease is an important risk factor for the development of TdP in line with information provided by CredibleMeds. ${ }^{10}$

In our study, the number of women and men who developed presumed drug-induced TdP were identical, despite women having higher TdP risk due to longer QTc intervals. ${ }^{1911}$ This finding may suggest that the TdP risk related to gender is of less importance compared with exposure to multiple TdP risk factors in our population of elderly people. This assumption is supported by hospital studies in patients with multiple risk factors treated with TdP-labelled drugs. Almost equal prevalence in LQTS/ $\mathrm{TdP}$ in women and men was observed; in two of them, a slightly increased risk in $\operatorname{men}^{27}{ }^{28}$ and in one a slightly increased risk in women ${ }^{24}$ were found. Overall, the results are in line with the concept of 'reduced repolarisation reserve', introduced by Roden, ${ }^{126}$ which emphasises that multiple risk factors are needed to overcome the complex and compensatory physiological mechanisms (reserve or redundancy) that interact to maintain a normal ventricular repolarisation. The high prevalence of prescription of TdP risk classified drugs in patients with additional risk factors, in this and other studies, ${ }^{27-31}$ also suggests that TdP induced by QTc-prolonging drugs is still a neglected issue for many physicians.

\section{TdP in relation to therapeutic groups and individual drugs}

Our results show that TdP 1-classified drugs overall were used more often than TdP 2-classified and TdP 3-classified drugs at the index event. The most used drugs at the index event were antidepressants followed by antiarrhythmic drugs, antipsychotics, urological drugs and drugs used to treat infections (antibiotics and fungicides). Antiarrhythmic drugs, classified with TdP 1 risk, showed the highest number of TdP per 100000 elderly users. The highest incidences were observed for disopyramide, followed by sotalol and amiodarone. These results seem to suggest that antiarrhythmic drugs have the highest torsadogenic potential among all therapeutic classes. These drugs are, however, prescribed mainly by cardiologists, who most likely are aware of the TdP risk. In that direction points a tendency towards lower concomitant use of other TdP-classified drugs (especially other TdP 1-classified or TdP 2-classified drugs) if the person was using an antiarrhythmic drug, compared with when TdP 1-classified antidepressants, antipsychotics or antidementia drugs were used.

Use of antidepressant drugs was associated with highest absolute numbers of TdP. This result indicate that use of TdP-labelled antidepressant drugs is a major contributing factor to observed TdP in older persons. Citalopram alone, with known TdP risk (TdP 1), was associated with a number of TdPs (72 cases) equal to the number of TdPs with all antiarrhythmic drugs together (71 cases). Citalopram had 2-4 times higher incidence of TdP per 100000 users during the study period, in both age groups, than the other widely used antidepressant drugs mirtazapin with possible (TdP 2) risk and sertraline with conditional (TdP 3) risk. Altogether, each of these antidepressants was prescribed to more than 700000 persons aged 18 years or older during the study period. Citalopram has also in other studies been associated with a higher risk for TdP than other antidepressant drugs. ${ }^{31}{ }^{32}$ In 2012 the EU and US regulatory authorities therefore issued a warning regarding use of citalopram at doses $>20 \mathrm{mg}$ in elderly and in persons with additional risk factors such as cardiac disease, electrolyte changes or use of other TdP-classified drugs. ${ }^{56}$ The results in the present study indicate that the adherence to these recommendations is low.

\section{TdP incidence: 'iceberg' problem}

Previous studies indicate that LQTS and TdP are underreported and that data on the incidence of $\mathrm{TdP}$ are scarce. ${ }^{243}$ There are several reasons for why it is difficult to define the true incidence of TdP or TdP-related death. One reason is that ECG monitoring at hospitals is necessary to establish the diagnosis of TdP. In addition, TdP and other ventricular arrhythmias, as well as sudden cardiac death, may not be properly identified even at hospitals, ${ }^{34}$ because many patients are not under continuous ECG surveillance. Moreover, a sizeable proportion of patients with TdP will not survive the ventricular arrhythmia. For example, in Sweden, $65 \%$ of all deaths occur outside the hospital setting. Yet another reason is probably a low reporting rate by healthcare professionals. In general, only a few per cent of adverse drug reactions (ADRs) are reported to the competent authorities; for example, only $1 \%$ of hospitalisations caused by ADRs are reported in the Netherlands. ${ }^{21}$ It is for the abovementioned reasons that it is very difficult to retrospectively make a TdP diagnosis, even if suspicions may exist; for example, an older woman treated with a drug with known TdP risk suddenly dies at home in connection with a gastroenteritis (which can cause electrolyte changes). Thus, our patient cohort most likely represents the tip of an iceberg.

\section{TdP incidence: all-cause death as a proxy}

All-cause death, adjusted for a number of potential comorbidity confounders, has been used as an outcome proxy to investigate the risk of TdP-related death in elderly persons prescribed TdP risk classified antipsychotics or antidepressants. ${ }^{13-17}$ In a previous Swedish 
nationwide study, ${ }^{17}$ we showed a stronger association between use of antidepressants classified with higher TdP risk (TdP 1 and TdP 2) and increased mortality in the elderly, compared with use of drugs without TdP labelling or with conditional TdP risk (TdP 3). The analysis was adjusted for several confounders, including education, number of inpatient days, contacts with open specialised healthcare, total number of drugs used, number of other drugs with TdP liability and a range of other potential comorbidity confounders. Persons aged $\geq 65$ years using antidepressants with known TdP 1 risk $(85 \%$ used citalopram) had an increased risk of premature death (OR 1.53), compared with those using antidepressants with conditional TdP 3 risk (82\% used sertraline or amitriptyline, OR 1.25). Non-users and users of antidepressants without TdP classification were used as reference (OR 1.0 ). The results of that study indicate (by calculation of attributable fraction) that the premature deaths among elderly users of antidepressants could have been reduced with 14\% if TdP 3-classified instead of TdP 1-classified antidepressants had been used, translated to more than 22000 deaths during the study period (2006-2013). Even if the results in that study cannot establish a causal relationship between TdP category and death, they indicate an increased TdP risk with the use of citalopram, in line with the present study.

The present study shows that use of citalopram was associated with a two to four times times increased incidence of TdP in both age groups, compared with TdP 3-classified sertraline and amitriptyline users. The results support that the TdP risk classification of antidepressants should be taken into consideration when prescribing to older people.

\section{TdP risk reduction}

Our results stress the need for initiatives to improve the prevention of TdP events in the future. Educational tools focusing on the awareness, knowledge and competencies of healthcare professionals regarding QTc prolongation and TdP need to be developed. Because of the high number of risk factors and the complexity of this risk, education should be combined with the implementation of advanced tools in daily practice, such as electronic clinical decision support systems. Such systems can create patient-specific alerts that automatically take into account the individual risk factors of the patient. These alerts can make the risk management of QTc prolongation and TdP more feasible, and closer monitoring can be limited to high-risk patients, as discussed by Vandael $e t$ al. ${ }^{21}$

\section{Limitations}

Sweden has excellent conditions for postmarketing surveillance of ADR. The collection of register data is governed by Swedish legislation, and it is mandatory for healthcare and pharmacies to report data. By combined analyses of different national registers with high coverage, our study can circumvent shortcomings of many other studies based on small and selected samples of individuals.
However, it must be emphasised that our study has several limitations. SPDR does not include information about drug treatment at hospitals. This is a major limitation since studies ${ }^{24} 34$ indicate that many TdPs are related to drug treatment at hospitals. Furthermore, we specifically focused on cases that were coded with the term 'TdP' (ICD code I47.2), while some TdP cases might have been classified under broader terms. Finally, a general limitation related to drug register data is that they may not fully reflect the patients' actual drug use, if adherence to treatment is low.

In conclusion, the results of the present investigation corroborate the results from other studies showing that TdP usually is the result of multiple risk factors, such as advanced age, use of more than one TdP-classified hERG-blocking drug, cardiovascular disease and possible electrolyte changes related to use of diuretics and/ or acid-secretion inhibitors. The study shows that antidepressants, particularly citalopram with known TdP risk (TdP 1), were associated with the highest absolute numbers of TdP, indicating that use of antidepressant drugs is a major contributing factor to TdP. The results further underscore that TdP risk should be taken into consideration when prescribing to older people and that the awareness, knowledge and competence of healthcare professionals regarding QTc prolongation and TdP need to be improved.

Acknowledgements We thank Alistair Hind at the Swedish National Board of Health and Welfare for review and correction of spelling and grammar errors.

Contributors BD, NB, MS and JF were involved in the conception, design and planning the study. BD, JC, AN, AB, LB and JF were responsible for acquisition of data and data analysis. $B D$ wrote the first draft. All authors participated in the interpretation of the results, contributed to further drafts and approved the final manuscript.

Funding This study had no funding, but was carried out as part of an assignment at the Swedish National Board of Health and Welfare.

Competing interests None declared.

Patient consent for publication Not required.

Ethics approval The study was performed within the responsibilities of the National Board of Health and Welfare and, therefore, no ethical approval from outside ethics committees was needed.

Provenance and peer review Not commissioned; externally peer reviewed.

Data availability statement № data are available. No original data are available, as they are individual-based and therefore protected.

Open access This is an open access article distributed in accordance with the Creative Commons Attribution Non Commercial (CC BY-NC 4.0) license, which permits others to distribute, remix, adapt, build upon this work non-commercially, and license their derivative works on different terms, provided the original work is properly cited, appropriate credit is given, any changes made indicated, and the use is non-commercial. See: http://creativecommons.org/licenses/by-nc/4.0/.

ORCID iD

Johan Fastbom http://orcid.org/0000-0001-9656-4668

\section{REFERENCES}

1 Roden DM. Drug-Induced prolongation of the QT interval. N Engl J Med 2004;350:1013-22.

2 Cohagan B, Brandis D. Torsades de pointes (Internet). StatPearls Publishing, 2019. https://www.ncbi.nlm.nih.gov/books/NBK459388/ 
3 Credible Meds - Available TdP risk categories. Arizona center for education on research on therapeutics. Available: https://www. crediblemeds.org/ [Accessed 15 Oct 2019].

4 Nachimuthu S, Assar MD, Schussler JM. Drug-Induced QT interval prolongation: mechanisms and clinical management. Ther Adv Drug Saf 2012;3:241-53.

5 U.S. Food \& Drug. FDA drug safety communication: revised recommendations for Celexa (citalopram hydrobromide) related to a potential risk of abnormal heart rhythms with high doses, 2012. Available: http://www.fda.gov/Drugs/DrugSafety/ucm297391.htm

6 European medicines agency. Pharmacovigilance Working Party (PhVWP) October 2011 plenary meeting. EMA/CHMP/ PhWWP/845939/2011. Available: http://www.ema.europa.eu/docs/ en_GB/document_library/Report/2011/10/WC500117061.pdf

7 Warnier M, Rutten F, Souverein P, et al. Are ECG monitoring recommendations before prescription of QT prolonging drugs applied in daily practice? The example of haloperidol. Pharmacoepidemiol Drug Saf 2014;23:228-9.

8 Vandael E, Vandenberk B, Vandenberghe J, et al. Risk factors for QTc-prolongation: systematic review of the evidence. Int J Clin Pharm 2017;39:16-25.

9 Hasnain M, Vieweg WVR. QTc interval prolongation and torsade de pointes associated with second-generation antipsychotics and antidepressants: a comprehensive review. CNS Drugs 2014;28:887-920.

10 Credible Meds www. Clinical factors associated with prolonged QTC and/or23 TdP. Arizona Center for Education on Research on Therapeutics, 2018. www.QTFactors.org

11 Wenzel-Seifert K, Wittmann M, Haen E. QTc prolongation by psychotropic drugs and the risk of torsade de pointes. Dtsch Arztebl Int 2011;108:687-93.

12 Lazzerini PE, Bertolozzi I, Finizola F, et al. Proton pump inhibitors and serum magnesium levels in patients with torsades de pointes. Front Pharmacol 2018;9:363.

13 Huybrechts KF, Gerhard T, Crystal S, et al. Differential risk of death in older residents in nursing homes prescribed specific antipsychotic drugs: population based cohort study. BMJ 2012;344:e977.

14 Leonard CE, Freeman CP, Newcomb CW, et al. Antipsychotics and the risks of sudden cardiac death and all-cause death: cohort studies in Medicaid and Dually-Eligible Medicaid-Medicare beneficiaries of five states. J Clin Exp Cardiolog 2013;10:1-9.

15 Kales HC, Kim HM, Zivin K, et al. Risk of mortality among individual antipsychotics in patients with dementia. Am J Psychiatry 2012;169:71-9.

16 Coupland C, Dhiman P, Morriss R, et al. Antidepressant use and risk of adverse outcomes in older people: population based cohort study. BMJ 2011;343:d4551.

17 Danielsson B, Collin J, Jonasdottir Bergman G, et al. Antidepressants and antipsychotics classified with torsades de pointes arrhythmia risk and mortality in older adults - a Swedish nationwide study. $\mathrm{Br} \mathrm{J}$ Clin Pharmacol 2016;81:773-83.
18 Ludvigsson JF, Andersson E, Ekbom A, et al. External review and validation of the Swedish national inpatient register. BMC Public Health 2011;11:450.

19 Wallerstedt SM, Fastbom J, Johnell K, et al. Drug treatment in older people before and after the transition to a Multi-Dose drug dispensing System-A longitudinal analysis. PLoS One 2013;8:e67088.

20 Öppna jämförelser. Läkemedelsbehandlingar, jämförelser mellan landsting. Socialstyrelsen, 2013: 6-13.

21 Vandael E, Vandenberk B, Vandenberghe J, et al. Cases of druginduced torsade de pointes: a review of Belgian cases in the EudraVigilance database. Acta Clin Belg 2017:72385-90.

22 Blaschke D, Parwani AS, Huemer M, et al. Torsade de pointes during combined treatment with risperidone and citalopram. Pharmacopsychiatry 2007:40:294-5.

23 Stella F, Loureiro JC, Pais MV, et al. Safety limits of antidepressant use plus combinations: focus on cardiovascular function. Curr Drug Metab 2018;19:641-52.

24 Vandael E, Vandenberk B, Vandenberghe J, et al. Incidence of torsade de pointes in a tertiary hospital population. Int $J$ Cardiol 2017:243:511-5.

25 Elliott A, Mørk TJ, Højlund M, et al. QTc interval in patients with schizophrenia receiving antipsychotic treatment as monotherapy or polypharmacy. CNS Spectr 2018;23:278-83.

26 Roden DM. Predicting drug-induced QT prolongation and torsades de pointes. J Physiol 2016;594:2459-68.

27 Khan Q, Ismail M, Haider I, et al. QT interval prolongation in hospitalized patients on cardiology wards: a prospective observational study. Eur J Clin Pharmacol 2017;73:1511-8.

28 Fernandes FM, Silva EP, Martins RR, et al. QTc interval prolongation in critically ill patients: prevalence, risk factors and associated medications. PLoS One 2018;13:e0199028.

29 Moreno-Gutiérrez PA, Gaviria-Mendoza A, Cañón MM, et al. High prevalence of risk factors in elderly patients using drugs associated with acquired torsades de pointes chronically in Colombia. $\mathrm{Br} \mathrm{J}$ Clin Pharmacol 2016;82:504-11.

30 Franchi C, Ardoino I, Rossio R, et al. Prevalence and risk factors associated with use of QT-Prolonging drugs in hospitalized older people. Drugs Aging 2016;33:53-61.

31 Aström-Lilja C, Odeberg JM, Ekman E, et al. Drug-induced torsades de pointes: a review of the Swedish pharmacovigilance database. Pharmacoepidemiol Drug Saf 2008;17:587-92.

32 Tampi RR, Balderas M, Carter KV, et al. Citalopram, QTc prolongation, and torsades de pointes. Psychosomatics 2015;56:36-43.

33 Braillon A. Torsade de pointes: the tip of the iceberg. Int $J$ Cardiol 2018;257:115.

34 Drew BJ, Ackerman MJ, Funk M, et al. Prevention of torsades de pointes in hospital settings: a scientific statement from the American heart association and the American College of cardiology Foundation. J Am Coll Cardiol 2010;55:934-47. 